\title{
Working memory brain activity and capacity link MAOA polymorphism to aggressive behavior during development
}

\author{
T Ziermans ${ }^{1}$, I Dumontheil ${ }^{1}$, C Roggeman ${ }^{1}$, M Peyrard-Janvid ${ }^{2}$, H Matsson ${ }^{2}$, J Kere ${ }^{2,3,4}$ and T Klingberg ${ }^{1}$
}

A developmental increase in working memory capacity is an important part of cognitive development, and low working memory (WM) capacity is a risk factor for developing psychopathology. Brain activity represents a promising endophenotype for linking genes to behavior and for improving our understanding of the neurobiology of WM development. We investigated gene-brainbehavior relationships by focusing on 18 single-nucleotide polymorphisms (SNPs) located in six dopaminergic candidate genes (COMT, SLC6A3/DAT1, DBH, DRD4, DRD5, MAOA). Visuospatial WM (VSWM) brain activity, measured with functional magnetic resonance imaging, and VSWM capacity were assessed in a longitudinal study of typically developing children and adolescents. Behavioral problems were evaluated using the Child Behavior Checklist (CBCL). One SNP (rs6609257), located $\sim 6.6 \mathrm{~kb}$ downstream of the monoamine oxidase $A$ gene (MAOA) on human chromosome $\mathrm{X}$, significantly affected brain activity in a network of frontal, parietal and occipital regions. Increased activity in this network, but not in caudate nucleus or anterior prefrontal regions, was correlated with VSWM capacity, which in turn predicted externalizing (aggressive/oppositional) symptoms, with higher WM capacity associated with fewer externalizing symptoms. There were no direct significant correlations between rs 6609257 and behavioral symptoms. These results suggest a mediating role of WM brain activity and capacity in linking the MAOA gene to aggressive behavior during development.

Translational Psychiatry (2012) 2, e85; doi:10.1038/tp.2012.7; published online 28 February 2012

\section{Introduction}

Working memory (WM) refers to the retention and manipulation of information over a brief period of time. ${ }^{1} \mathrm{~A}$ gradual increase in WM capacity is strongly related to the development of general intellectual ability ${ }^{2}$ and academic performance. ${ }^{3}$ Impaired WM capacity is a core feature of many psychiatric disorders, including attention-deficit/hyperactivity disorder ${ }^{4}$ and schizophrenia. ${ }^{5}$ Furthermore, low WM capacity in children is a risk factor for psychopathology later in life, such as psychosis, depression and suicidal ideation. ${ }^{6,7}$ WM capacity is also an important factor for emotional selfregulation, ${ }^{8}$ and restricted self-regulation has been associated with behavioral problems in children, in particular, but not restricted to, externalizing problems (for example, aggression, antisocial behavior). ${ }^{9}$ Increasing knowledge of the underlying neurobiological mechanisms of typical WM development is an essential step to understand atypical development and guide initiatives to remediate problem behavior. ${ }^{10}$

Genetic effects on psychiatric phenotypes are often subtle and hard to verify, possibly due to the interindividual heterogeneity of the phenotype. An alternative approach is to focus on the functional deficits associated with psychiatric disorders, such as WM impairments.
However, these cognitive endophenotypes ${ }^{11,12}$ are the results of interactions between multiple brain regions. An interesting possibility is that biological measures, such as brain structure and function, ${ }^{13}$ which are biologically more closely linked to gene activity, may be more useful endophenotypes, with better potential of finding genetic associations. $^{11}$

Functional magnetic resonance imaging (fMRI) studies have consistently shown WM-related blood-oxygen level dependent (BOLD) activations in a fronto-parietal network of brain regions, particularly in visuospatial WM (VSWM) paradigms. ${ }^{14}$ This fronto-parietal activity increases with age from childhood to young adulthood ${ }^{15-19}$ and correlates with accuracy on a trial-by-trial basis. ${ }^{17,20,21}$ Although some specific genetic polymorphisms have been associated with individual differences in capacity during this period, ${ }^{22,23}$ the genetic basis for WM capacity is still largely unknown. Furthermore, the behavioral consequences of WM-related brain activity, and its association with psychopathological symptoms in particular, have not been reported previously. Therefore, in light of the onsets of certain psychopathologies during childhood and adolescence, elucidating the nature of gene-brain-behavior relationships during development seems critical.

\footnotetext{
${ }^{1}$ Neuroscience Department, Karolinska Institutet, Stockholm, Sweden; ${ }^{2}$ Department of Biosciences and Nutrition at Novum, Karolinska Institutet, Huddinge, Sweden; ${ }^{3}$ Science for Life Laboratory, Department of Biosciences and Nutrition at Novum, Karolinska Institutet, Solna, Sweden and ${ }^{4}$ Department of Medical Genetics, Haartman Institute, 00014 University of Helsinki, Helsinki, Finland

Correspondence: Dr T Ziermans, Department of Neuroscience, Retzius väg 8, Karolinska Institutet, 17177 Stockholm, Sweden.
}

E-mail: tim.ziermans@ki.se

Keywords: CBCL; dopamine; fMRI; MAOA; single nucleotide polymorphism; working memory

Received 22 December 2011; accepted 8 January 2012 
On a molecular level, dopamine (DA) availability affects VSWM function, as demonstrated by experiments in both humans ${ }^{24}$ and primates. ${ }^{25}$ It has been suggested that DA regulation of VSWM is subject to developmental changes before adulthood, although for humans there is only indirect evidence from molecular genetic studies. ${ }^{26}$ Furthermore, performance-related differences in regional brain activity may be partially due to differences in DA signaling in those regions. ${ }^{27,28}$ Genes involved in regulation of the DA system are therefore of particular interest, especially candidate genes linked to psychiatric disorders in which WM functioning is typically impaired. ${ }^{29,30}$

In the present study, we aimed to investigate VSWMrelated brain activity and VSWM capacity as intermediary phenotypes between genetic variation of the DA system and problem behavior. We genotyped single-nucleotide polymorphisms (SNPs) in and in near vicinity of six genes implicated in DA transmission: COMT, SLC6A3/DAT1, DBH, $D R D 4, D R D 5$ and MAOA. Problem behavior was assessed using the Child Behavioral Checklist (CBCL). ${ }^{31}$ Higher CBCL scores have previously been associated with low WM performance in preadolescents ${ }^{32}$ and can predict future onset of psychopathology in children. ${ }^{33}$ It was hypothesized that BOLD activity in a fronto-parietal brain network would increase during development and be positively associated with VSWM capacity. Furthermore, we expected that polymorphisms in genes of the DA system would affect brain activity in this network and that these genetic effects may be age dependent. ${ }^{22}$ Finally, we hypothesized that VSWM capacity would predict CBCL scores in a large regional cohort of Swedish children and young adults and, in addition, tested whether genotypes of the DA system genes affecting VSWM brain activity could predict VSWM capacity and CBCL scores.

\section{Methods}

Participants. A total of 335 participants from nine different age groups $(6,8,10,12,14,16,18,20$ and 25 years) were randomly selected out of a community sample from Nynäshamn in Sweden ('Brainchild' study). ${ }^{22,23}$ Number of participants was distributed evenly among the different age groups (45 on average), except for the two oldest age groups, including 13 and 8 participants, respectively. Assessments included a baseline measurement (round 1) and a second round of testing 2 years later (round 2). A large majority $(88.7 \%)$ of the participants and their parents were born in Sweden, 9.3\% had at least one parent born outside Sweden, although still within Europe, and the remaining $2 \%$ had one or two parents that were born outside of Europe. All participants were enrolled in mainstream schools. Exclusion criteria were first language other than Swedish, diagnosis of neurological or psychiatric disorder (with exception of attention-deficit/hyperactivity disorder or dyslexia). Informed consent was obtained from the participants and from the parents of children aged 18 years or younger. The local ethics committee of the Karolinska University Hospital, Stockholm, approved the study. Additional information on genotyping and participant exclusion can be found in the Supplementary Information.
Behavioral sample. Data on VSWM capacity was available for 329 participants; round 1: $n=329$ (49.2\% male), 12.2 years \pm 4.5 (mean age \pm s.d.), round $2: n=268 \quad(50.4 \%$ male), 13.6 years \pm 4.0 . Data on problem behavior was available for participants of age 18 years and younger; round 1: $n=260$ (49.2\% male), 11.0 years \pm 3.3 , round $2: n=233$ (50.2\% male), 12.7 years \pm 3.3 . All participants at round 2 had participated in round 1 as well and therefore represented repeated measures.

fMRI sample. Eighty nine participants were randomly selected from all age groups of the behavioral sample to participate in $\mathrm{fMRI}$ assessments. In total, 134 measurements were included; round $1: n=68(51.5 \%), 13.8$ y \pm 5.3 , round 2 : $n=66$ (43.9\%), $14.9 \mathrm{y} \pm 4.7$ (including 45 repeated measures of round 1 participants).

Genetic analysis. Material for DNA extraction was collected in form of blood or saliva. Genotyping procedures have been previously published. ${ }^{22,23}$ Details of the 18 SNPs used for this study are available in Table 1. For every SNP, two independent scorers confirmed all genotypes, and regenotyping of $5 \%$ of the study samples resulted in $100 \%$ concordance. All markers were found to be in Hardy-Weinberg equilibrium, taking autosomal or sexchromosomal localization of the markers into account.

\section{fMRI}

Scan protocol. Subjects were positioned head first and supine in the MR scanner. Images were collected using a 1.5T Siemens Avanto scanner (Siemens Medical Systems, Erlangen, Germany), using an 8-channel radio frequency head coil. For the structural scan, 19 slices were acquired using a $\mathrm{T} 1$-weighted $3 \mathrm{D}$ anatomical sequence $(\mathrm{TR}=308 \mathrm{~ms}, \quad \mathrm{TE}=7.8 \mathrm{~ms}$, image matrix $=256 \times 256$,

Table 1 Dopaminergic genes and genotyped SNPs

\begin{tabular}{|c|c|c|c|}
\hline $\begin{array}{l}\text { Gene } \\
\text { symbol }\end{array}$ & Gene name & $\begin{array}{l}\text { Chromosome } \\
\text { Band }^{\text {a }}\end{array}$ & $S N P$ \\
\hline DRD5 & Dopamine receptor D5 & $4 p 16.1$ & $\begin{array}{l}\text { rs13140817 } \\
\text { rs10939515 } \\
\text { rs1967550 }\end{array}$ \\
\hline $\begin{array}{l}\text { SLC6A3 } \\
\text { (DAT1) }\end{array}$ & $\begin{array}{l}\text { Solute carrier family } 6 \\
\text { member } 3 \text { (dopamine } \\
\text { transporter) }\end{array}$ & $5 p 15.3$ & $\begin{array}{l}\text { rs3863145 } \\
\text { rs27072 }\end{array}$ \\
\hline$D B H$ & Dopamine beta-hydroxylase & $9 q 34$ & $\begin{array}{l}\text { rs1611125 } \\
\text { rs1541332 } \\
\text { rs2797853 }\end{array}$ \\
\hline$D R D 4$ & Dopamine receptor D4 & $11 p 15.5$ & $\begin{array}{l}\text { rs7124601 } \\
\text { rs11246226 } \\
\text { rs936465 }\end{array}$ \\
\hline COMT & $\begin{array}{l}\text { Catechol-O- } \\
\text { methyltransferase }\end{array}$ & $22 q 11.2$ & $\begin{array}{l}\text { rs740601 } \\
\text { rs4680 }\end{array}$ \\
\hline MAOA & Monoamine oxidase A & Xp11.3 & $\begin{array}{l}\text { rs5905702 } \\
\text { rs5906957 } \\
\text { rs2283724 } \\
\text { rs979606 } \\
\text { rs6609257 }\end{array}$ \\
\hline
\end{tabular}

Abbreviation: SNP, single-nucleotide polymorphism.

${ }^{a}$ Chromosome band according to Entrez Gene and NCBI Map Viewer, NCBI Build 36.3. 
FOV $=230 \mathrm{~mm}$, flip angle $=90^{\circ}$, slice thickness $=5.0 \mathrm{~mm}$, voxel size $=1.0 \times 0.9 \times 5.0 \mathrm{~mm}$ ). Whole-brain functional images were collected using a T2*-weighted EPI sequences, sensitive to BOLD contrast $(T R=3000 \mathrm{~ms}, T E=50 \mathrm{~ms}$, image matrix $=64 \times 64, F O V=220 \mathrm{~mm}$, flip angle $=90^{\circ}$, slice thickness $=4.5 \mathrm{~mm}$, distance factor $=11 \%$, voxel size $3.4 \times 3.4 \times 4.5 \mathrm{~mm}, 30$ axial slices). Each functional MRI run consisted of 96 images, preceded by two dummy scans that were excluded from further analysis.

VSWM paradigm. Participants performed two sessions of the task, each with a total of $16 \mathrm{WM}$ and 16 control trials, in a pseudo-randomized order. Dots were presented sequentially in a four-by-four grid for $500 \mathrm{~ms}$, with $500 \mathrm{~ms}$ interval between dots. The task included $50 \%$ load 2 and $50 \%$ load 4 trials. After $1500 \mathrm{~ms}$ delay, a number cue referring to a serial position in the previous stimulus sequence was presented in the grid for $3000 \mathrm{~ms}$. Participants indicated with a yes/no (right index/middle finger) response whether the number and its position in the grid matched. In the control condition, the number cue was 8 and the response was always no, regardless of the position of the question mark. Control and WM trials were indicated by the color of the dots (red: WM trial, yellow: control trial). The response screen remained visible for $3 \mathrm{~s}$, and was followed by a $2 \mathrm{~s}$ inter-trial interval. Total duration was $8 \mathrm{~s}$ for load 2 and $10 \mathrm{~s}$ for load 4 trials. The session started with a $6 \mathrm{~s}$ blank screen, resulting in a total duration of $4 \mathrm{~min}, 54 \mathrm{~s}$ for each session. Task, protocol and scanning parameters were exactly the same at both time points.

Data processing. Pre-processing and statistical analyses were carried out with SPM5 (www.fil.ion.ucl.ac.uk/spm/ software/spm5) and have previously been published. ${ }^{22,23}$ Runs were checked separately and therefore subjects could have one excluded run, and still be included with only one functional run. Functional images were first corrected for slice timing. Motion during scanning was then estimated by six parameters (three translations, three rotations) by minimizing the differences between the first volume and the subsequent volumes. These parameters were then used to realign the functional volumes to the first image of each run (second-degree B-spline interpolation). The mean image of the realigned functional images was co-registered to the anatomical scan, and the estimated co-registration parameters were then used to co-register all functional images. Next, the anatomical image was normalized to the Montreal Neurological Institute template, and the estimated normalization parameters were used to normalize all functional images. Finally, functional images were highpass (140 s) filtered and smoothed with a Gaussian kernel of $12 \mathrm{~mm}$ full-width half maximum prior to statistical analysis.

Activations were modeled for each subject separately using a general linear model analysis with four predictors. The predictors were built by convolving the actual onset times of the experimental events with the standard hemodynamic response function. The four experimental events consisted of the correct trials only: working memory trials with load 2 and load 4, and control trials with load 2 and load 4 . The duration of the events was set to include the time of maintenance and response, and was therefore equal to $8 \mathrm{~s}$ in load 2 trials and
$10 \mathrm{~s}$ in load 4 trials. Time and dispersion derivatives, as well as the motion parameters and the global mean, were added in the design. With this model, one contrast image, 'WM versus control', was made for each subject by contrasting both working memory conditions with both control conditions.

These 'WM versus control' contrast images were used in flexible factorial design second-level analyses, which modeled whether the contrasts were from the same or different participants by including subject and testing round as factors. Regions of interest (ROI) based on local maxima were defined and extracted using MarsBaR. ${ }^{34}$ First, ROIs were created based on all activations from the contrast 'WM versus control', thresholded at FDR (false discovery rate) $P<0.000001$. These ROIs were then split into smaller regions, so that each $\mathrm{ROI}$ was centered around a single local maximum. ROls were defined so that individual regions did not overlap, and together covered the majority of the working memory activation. Resulting ROls were plotted on a surface-based human atlas (PALS) $^{35,36}$ using the Caret software ${ }^{37}$ (www.nitrc.org/projects/caret/). For each ROI, the average WM-control BOLD of all voxels within that $\mathrm{ROI}$ was calculated for each participant.

\section{Behavioral assessment}

VSWM capacity. Participants completed a large neuropsychological battery administered individually. VSWM capacity was assessed using a visuospatial grid task (dot matrix) from the AWMA battery ${ }^{38}$ similar to the VSWM fMRI paradigm (see refs 22, 23 for a detailed description).

Problem behavior. Parental reports were used to assess behavioral problems in 4-18-year-old children on the CBCL. ${ }^{31,39}$ Three broadband scales (which assess a wide variety of behavioral symptoms) were used: total problems and the two subscales internalizing and externalizing problems. These last two scales largely correspond to mood/anxiety and disruptive behavior disorders, respectively.

Statistical analyses. Data were analyzed with linear mixed models, using the PASW 18.0 statistical package..$^{22,40}$ This approach allows all data to be included within a single model, even when some participants have only attended one testing session. A compound symmetry covariance structure was used, one parameter corresponding to the variance within sessions, and the other to the covariance between sessions. In the fMRI sample, BOLD activity and VSWM capacity were entered as dependent variables in separate analyses. In the behavioral sample, VSWM capacity and problem behavior (CBCL T-scores) were used as dependent measures in separate analyses. Effects were considered significant at the level of $P<0.05$. Bonferroni-corrected $P$-values were applied when testing for genetic effects.

\section{Results}

\section{fMRI}

In-scanner performance. Overall accuracy was high, but participants showed lower accuracy in the WM than the control condition (WM: mean 90.0\% \pm 11.0 ; control: $98.3 \% \pm 3.5)(\mathrm{t}(134)=10.15, P<0.001)$, as well as slower 
reaction time (correct trials only, WM: $1272 \mathrm{~ms} \pm 352$; control: $737 \mathrm{~ms} \pm 212) \quad(\mathrm{t}(134)=26.11, \quad P<0.001)$. The difference between WM and control conditions decreased with age for both accuracy and reaction time (main effect of age: $\mathrm{F}(1,73.9)=14.95, P<0.001$, and $\mathrm{F}(1,90.3)=35.0, P<0.001)$.

Main effect WM-control. Based on activity in the main effect of WM minus control $(P<0.05$, FDR $)$, a total of 17 individual ROls were identified and delineated: 8 frontal, 4 parietal, 3 occipital and 2 in the bilateral caudate nucleus (see Table 2).

Principal component analysis (PCA). ROI data were split per assessment (round 1 and 2) and transformed into a Z-score. All bilateral ROls correlated higher with each other (all $>0.7$ ) than with other ROIs, and were collapsed to prevent multicollinearity in the data. In order to further reduce the number of comparisons, BOLD data for each ROI was entered into a PCA.

Factors were rotated using the direct oblimin method, assuming that underlying factors are not completely independent. An eigenvalue of 0.7 was used as cut-off for factor extraction and all communalities had to be 0.7 or higher after extraction. ${ }^{41}$ Initial PCA led to exclusion of the left cuneus ROI, due to a low communality (0.4). After removal of this ROI, the eight remaining ROls (see Table 2) were used for PCA. Two-, three- and four-factor solutions were explored. The three-factor solution (F1, F2, F3; see Figure 1 and Table 2) accounted for $80.7 \%$ of the total variance and was considered most optimal. Running PCA separately for round 1 and 2 resulted in the same factor structures for both time points.
Composite scores were created for each factor, based on the means of the ROIs with factor loadings $>0.5$ in the final pattern matrix (highlighted in bold values in Table 2).

VSWM activity, age, sex and VSWM capacity. The mean BOLD value for each factor was regressed against three possible Z-score-transformed age variables: age $\left(\mathrm{age}_{\mathrm{Z}}\right)$, the inverse of age $\left(\mathrm{age}_{\mathrm{z}}^{-1}\right)$, or the natural logarithm of age $\left(\right.$ In $\left.(\text { age })_{z}\right)$, using linear mixed models, to assess the best fit for developmental changes in VSWM-related BOLD activity. Sex was entered as a main effect and sex $\times$ age as an interaction effect. Age $\bar{z}^{-1}$ provided the best fit for all factors based on the Akaike's information criterion. ${ }^{42}$ There was a significant increase in BOLD activity for all three factors with age $(\mathrm{F} 1: F(1,84.6)=45.68, P<0.001 ; \mathrm{F} 2: F(1,88.5)=5.58$, $P=0.020$; F3: $F(1,84.9)=12.71, P=0.001$ ), (Figure 2a). There was an additional main effect of sex for F2 $(F(1,72.4)=4.70, P=0.033)$ with males showing higher activity levels than females. No significant sex $\times$ age interaction was observed.

All three BOLD factors were independently tested for an association with VSWM capacity. $\mathrm{Age}_{\mathrm{Z}}^{-1}$ and sex were included as main effects in the model. Only $\mathrm{F} 1$ was significantly predicted by VSWM capacity $(\mathrm{F} 1: F(1,111.6)=$ 8.72, $P=0.003$ ) and only when age $z=1$ was not included in the model (Figure 2b). No significant interactions between BOLD factors, $a e_{z}^{-1}$ or sex were observed. This showed that, although activity increased with age in all factors, only activation within $\mathrm{F} 1$ regions was associated with VSWM capacity during development.

Table 2 ROls for the main effect of working memory-control

\begin{tabular}{|c|c|c|c|c|c|c|}
\hline Region & Side & $M N I(x, y, z)$ & $F 1$ & $F 2$ & F3 & Communalities \\
\hline Superior parietal & $\stackrel{L}{R}$ & $\begin{array}{c}-16,-62,60 \\
22,-66,50\end{array}$ & 0.96 & -0.07 & 0.02 & 0.87 \\
\hline Inferior parietal & $\begin{array}{l}\mathrm{L} \\
\mathrm{R}\end{array}$ & $\begin{array}{c}-36,-40,42 \\
42,-38,48\end{array}$ & 0.87 & 0.07 & -0.01 & 0.84 \\
\hline Lateral occipital & $\stackrel{L}{R}$ & $\begin{array}{l}-34,-82,2 \\
36,-80,-2\end{array}$ & 0.84 & -0.01 & 0.08 & 0.75 \\
\hline Superior frontal & $\frac{\mathrm{L}}{\mathrm{R}}$ & $\begin{array}{l}-22,2,56 \\
26,4,52\end{array}$ & 0.65 & 0.26 & 0.15 & 0.76 \\
\hline Middle frontal gyrus & $\mathrm{R}$ & $40,32,28$ & 0.08 & 0.90 & -0.17 & 0.81 \\
\hline Inferior frontal gyrus & $\begin{array}{l}\mathrm{L} \\
\mathrm{R}\end{array}$ & $\begin{array}{l}-32,22,0 \\
32,24,0\end{array}$ & -0.17 & 0.77 & 0.37 & 0.78 \\
\hline Pre-SMA & $\mathrm{L} / \mathrm{R}$ & $-4,16,50$ & 0.10 & 0.74 & 0.14 & 0.73 \\
\hline Inferior frontal sulcus & $\frac{\mathrm{L}}{\mathrm{R}}$ & $\begin{array}{c}-42,6,32 \\
48,8,30\end{array}$ & 0.42 & 0.65 & -0.14 & 0.81 \\
\hline Caudate & $\begin{array}{l}\mathrm{L} \\
\mathrm{R}\end{array}$ & $\begin{array}{c}-16,-4,20 \\
20,-2,18\end{array}$ & 0.23 & 0.04 & 0.85 & 0.92 \\
\hline Cuneus $^{a}$ & $\mathrm{~L}$ & $-10,-84,2$ & na & na & na & na \\
\hline $\begin{array}{l}\text { Factor characteristics } \\
\text { Eigenvalue }\end{array}$ & & & 5.47 & 1.09 & 0.70 & \\
\hline$\%$ of variance & & & 60.8 & 12.1 & 7.8 & \\
\hline Correlations & & & 1 & $\begin{array}{c}0.545 \\
1\end{array}$ & $\begin{array}{c}0.249 \\
0.359 \\
1\end{array}$ & \\
\hline
\end{tabular}

Abbreviations: F1-F3, Factor 1-3; L, left; na, not applicable; pre-SMA, pre-supplementary motor area; R, right; ROI, region of interest.

aNot included in factor analysis.

Location of ROI peak values as well as factor loadings and communalities for variables used in factor analysis are displayed. Values in bold highlight the grouping of ROIs in the three factors F1, F2, F3 identified by principal component analyses. 


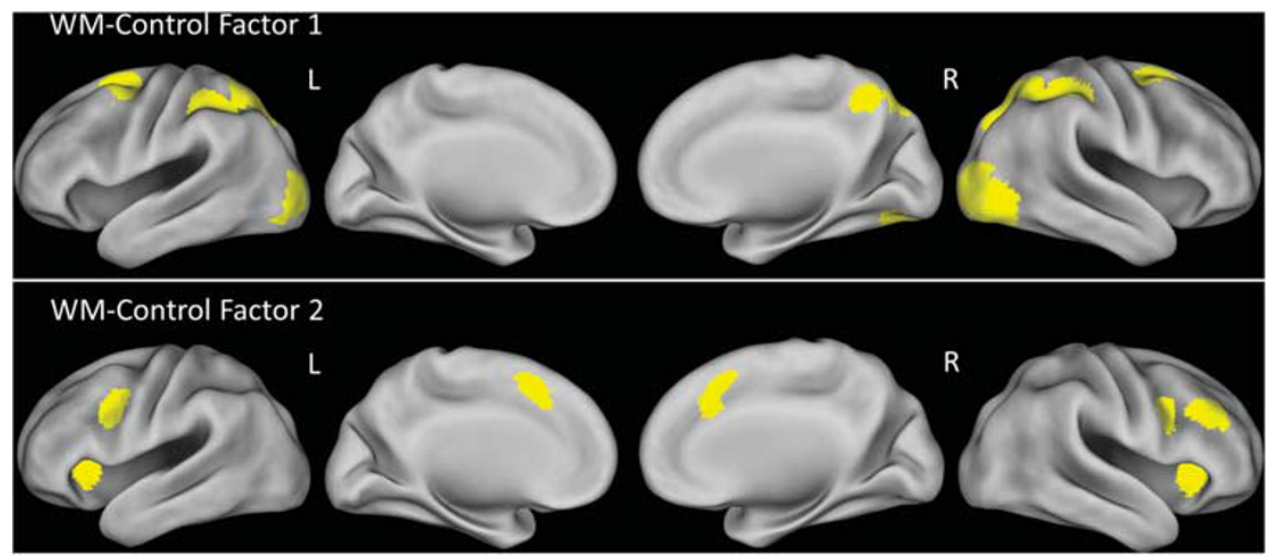

Figure 1 Render of delineated regions of interest (ROIs) of the (fMRI) main effect working memory (WM)-control (false discovery rate (FDR), $P<0.05)$ on a surface-based human atlas (see Methods). Top row: Factor 1 ROls with lateral and medial view of the left and right hemisphere. Bottom row: Factor 2 ROls with lateral and medial view of the left and right hemisphere.
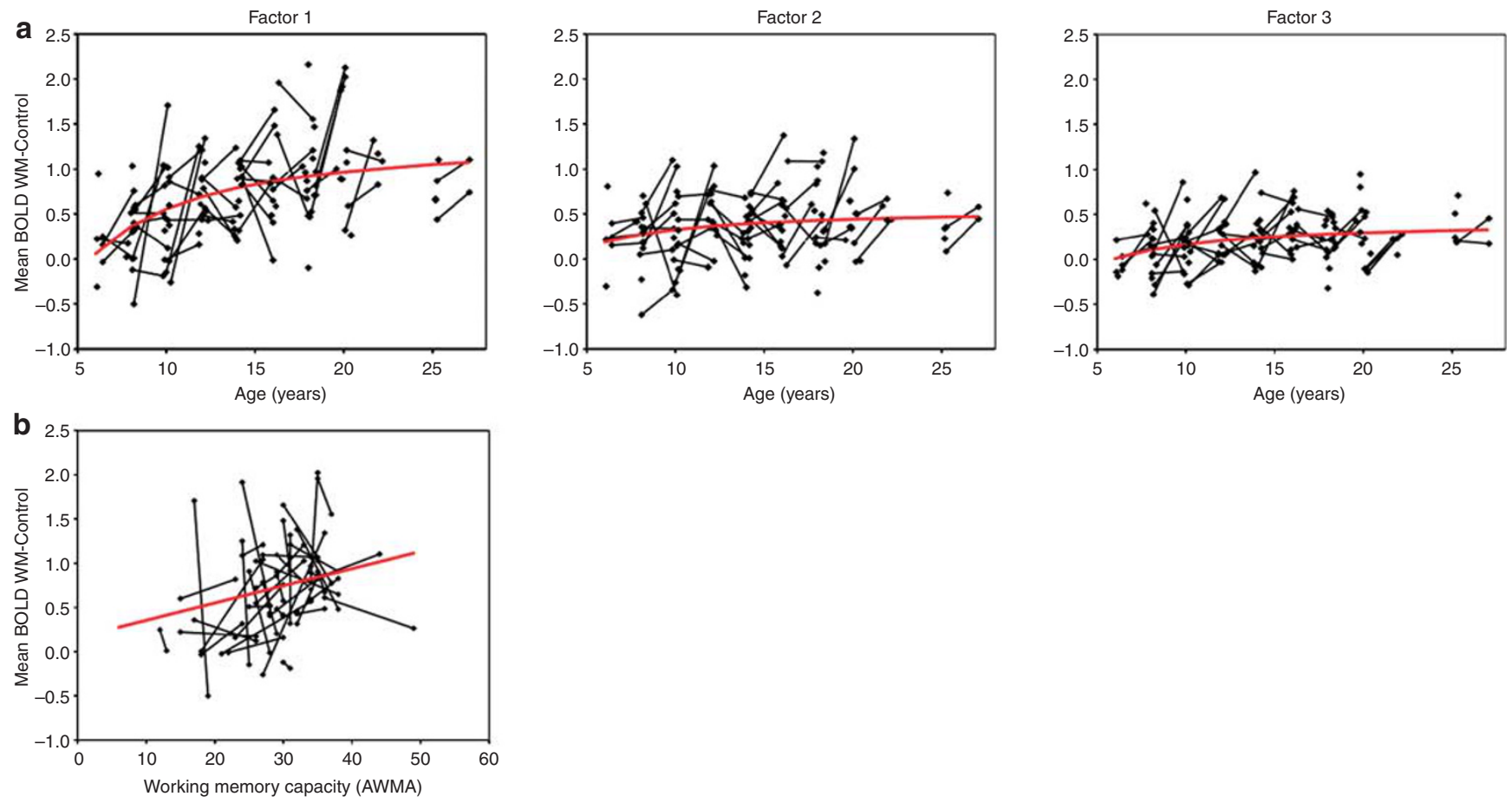

Figure 2 (a): Scatterplot of the mean blood-oxygen level dependent (BOLD) working memory (WM)-control values plotted against age for all three factors. Black lines connect repeated measures for individuals who participated in both round 1 and 2. Red lines represent the mean predicted BOLD signal as a function of age, as obtained from the mixed-model analysis using the inverse of age as a fixed effect. (b) Scatterplot of the mean BOLD WM-control values of Factor 1 against Visuospatial WM (VSWM) capacity. Black lines connect repeated measures. The red line represents the mean-predicted BOLD signal for each age group, as obtained from the mixed-model analysis using the inverse of age as a factor.

Genotypes and VSWM activity. Linear mixed models with age $_{z}^{-1}$, sex, genotype and genotype $\times$ age $_{z}^{-1}$ entered as covariates were used to test for genetic effects on VSWM-BOLD activity. Additive models were used for testing the effect of each genotype (for example, $0=A A, 1=A G$, $2=\mathrm{GG}$ for the rs4680 located in the COMT gene). Similar additive models were used for X-chromosomal SNPs located in the MAOA (monoamine oxidase A) gene by grouping men with homozygous females, rendering the heterozygote group $100 \%$ female. Any significant main or interaction effects for these SNPs were followed up by sex-specific post hoc tests. Due to a minor allele frequency $<10 \%$ within the fMRI sample, three SNPs (DRD5: rs10939515; SLC6A3/DAT1: rs27072 and rs3863145) were re-coded to pool the rare homozygotes with the heterozygotes. To limit the number of tests, only F1 was analyzed, as this was the only BOLD factor found to be associated with VSWM capacity.

Two of eighteen SNPs, rs979606 and rs6609257 (both located within or near the MAOA gene, see Figure 3 ), showed a main effect of genotype on $\mathrm{F} 1$, with only the latter surviving 


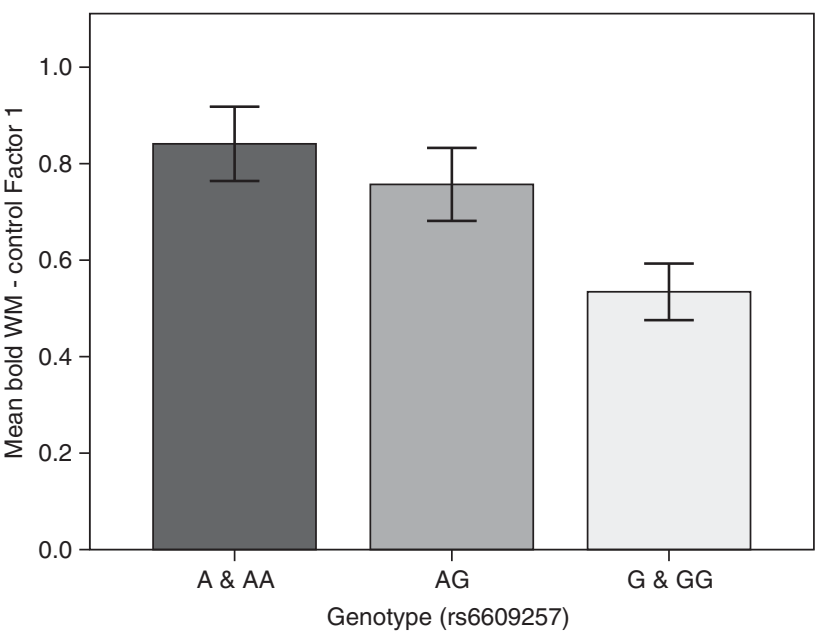

Figure 3 Factor 1 BOLD (blood-oxygen level dependent) working memory (WM)-control contrast displayed for the MAOA single-nucleotide polymorphism (SNP) rs6609257. This chart is included for illustrative purposes and represents the means and s.e.m. (collapsing across longitudinal and cross-sectional data) of F1 mean working memory (WM)-control BOLD signal. Participants tested in both rounds were therefore included as two data points. The size of each group of genotypes is: 60 (hemizygotes $A$ and homozygotes AA), 32 (heterozygotes AG) and 42 (hemizygotes $G$ and homozygotes $G G$ ). The heterozygote (AG) group only represents female participants, as the MAOA gene is located on the $\mathrm{X}$-chromosome. All hemizygote genotypes are from male participants who have either a single $A$ or $G$ allele.

Bonferroni correction for multiple comparisons $(F(1$, $75.7)=11.52, \quad P=0.001$ ). The same SNP (rs6609257, $A>G$ ) was the only one to show an interaction effect for genotype $\times$ age $_{z}^{-1}$, but this effect did not survive Bonferroni correction $(P=0.02)$. Mean-predicted values of $\mathrm{F} 1$ as a function of rs6609257 indicated that the A allele was associated with higher levels of VSWM-BOLD activity than the $G$ allele (Figure 4). No main effect of sex was found, and post hoc testing did not show any effects when interactions with sex were added to the model (that is, $\mathrm{age}_{\mathrm{z}}^{-1} \times$ sex, genotype $\times$ sex, age $z^{-1} \times$ sex $\times$ genotype). When the model was applied separately for males $(n=64)$ and females $(n=70)$, the main effect of genotype was significant for males, $F(1,35.4)=6.62, P=0.014$, with A males showing higher levels of WM activation than $G$ males. For females, there was a trend $(P=0.08)$ in the same direction (AA and AG versus $G G$ ).

MAOA, VSWM capacity and problem behavior. To test whether the identified MAOA SNP affecting VSWM-BOLD, rs6609257, also directly affected VSWM capacity in the behavioral sample, linear mixed models were used. $\mathrm{Age}_{\mathrm{Z}}^{-1}$, sex, genotype and genotype $\times$ age were entered in the model. There was a strong main effect of $\operatorname{age}_{\bar{z}}^{-1}(F(1,507.3)=233.80$, $P<0.001$ ), and no additional effect of sex, genotype or interaction effect of age $z^{-1} \times$ genotype on VSWM capacity. Further exploration of main and interaction effects after splitting the group for sex did not affect the outcome.

We next tested whether rs6609257 predicted problem behavior. Sex and age were excluded from the model because CBCL T-scores are corrected for these variables

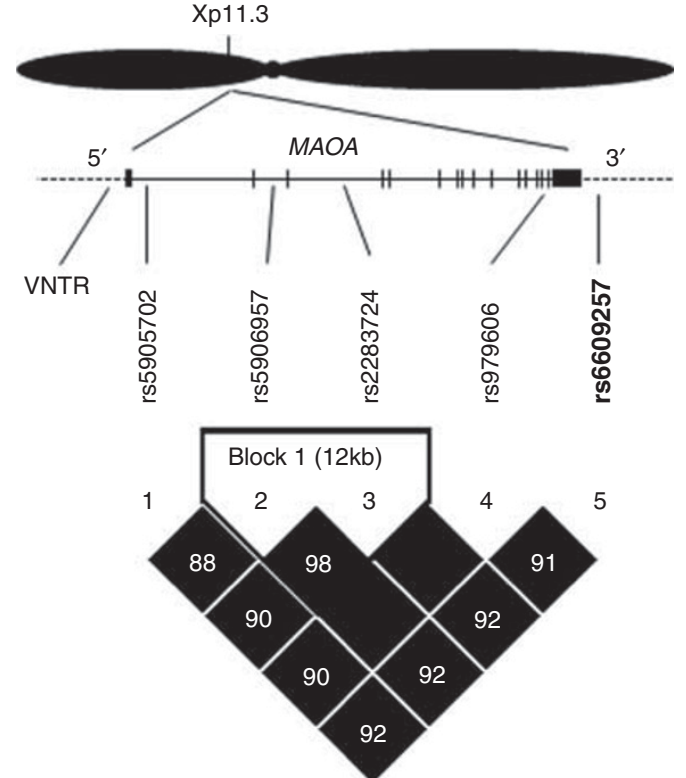

Figure 4 Chromosome $X$ region (top) containing the genotyped singlenucleotide polymorphisms (SNPs) with corresponding linkage disequilibrium (LD) values. The variable number of tandem repeats (VNTR) previously shown to regulate $M A O A$ activity is located approximately 1000 bases upstream of the MAOA transcription start site. Protein-coding exons are depicted as vertical lines and the $5^{\prime}$ and $3^{\prime}$ untranslated regions as filled black boxes. Rs6609257, significantly associated to visuospatial working memory (VSWM) after Bonferroni correction in this study, is highlighted in bold. The bottom panel shows the calculated LD between pairs of SNPs as percentage of the theoretical maximal LD with logarithm of likelihood odds ratio (D'/LOD) using Haploview 4.2. All SNPs are in high or complete LD with statistical significance. Values are based on our own 'Brainchild' sample set. Black squares without text depict complete (100\%) LD.

based on population norms. There was no main effect of genotype on total, internalizing or externalizing problems. Finally, we analyzed whether VSWM capacity was able to predict problem behavior when entered as a single fixed effect in the model. VSWM capacity significantly predicted level of externalizing problems $(F(1,492.3)=4.11, P=0.043)$, with low WM associated with more externalizing problems, and at trend-level for total problems $(P=0.08)$.

The main results of this study are combined and illustrated by means of a heuristic model in Figure 5.

\section{Discussion}

The aim of this study was to investigate the role of VSWM development, both in terms of behavior and brain activity, as an intermediate phenotype linking DA-related genes to problem behavior. VSWM-related brain activity in frontoparieto-occipital regions (Factor 1 ) increased during development and was positively associated with VSWM capacity. Brain activity in this network was modulated by genotype for one SNP, rs6609257, located $\sim 6.6 \mathrm{~kb}$ downstream of the MAOA gene on Xp11.23 (see Figure 3). On a behavioral level, we found that lower levels of externalizing problems were directly related to higher WM capacity.

MAO genes (MAOA and MAOB, separated by $19.8 \mathrm{~kb}$ on human chromosome $X$, in a head-to-head orientation) encode 


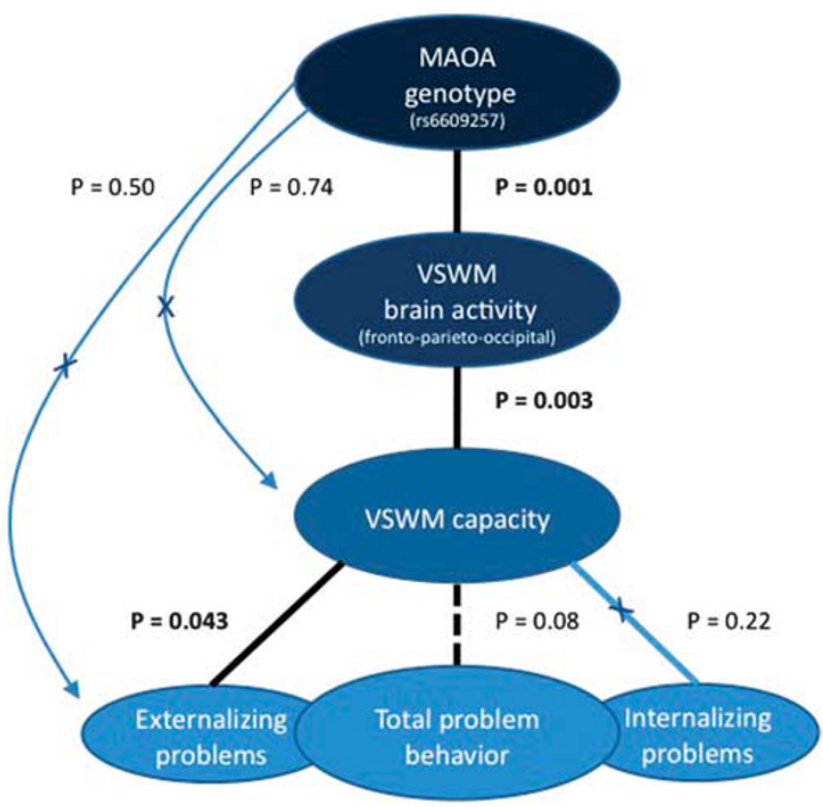

Figure 5 Heuristic hierarchical model summarizing the main findings in our study. Solid black lines represent significant associations, the dashed black line indicates a trend-level association and blue lines marked with a cross refer to nonsignificant associations. Relevant $P$-values are provided adjacent to each line in the model with significant effects printed in bold lettertype.

enzymes that are expressed throughout the brain and affect multiple monoaminergic systems by degrading DA, norepinephrine and serotonin. ${ }^{43}$ Previous studies have highlighted potential modulating effects of an MAOA variable number of tandem repeats (VNTR) in the MAOA promoter region (MAOA VNTR 30-bp repeat) with high- and low-activity variants. ${ }^{44-47}$ This VNTR and a previously identified haplotype of three MAOA polymorphisms (rs12843268, rs3027400 and rs1137070) have also been linked specifically to VSWM cognition and brain activity. ${ }^{48,49}$ Furthermore, on a behavioral level, the MAOA VNTR has been associated with traits such as depression ${ }^{50}$ and aggression, ${ }^{51}$ although ambiguously. ${ }^{52-54}$ Our study is consistent with the involvement of MAOA polymorphisms in regulating neurobiological mechanisms associated with VSWM capacity, which in turn can predict maladaptive behavior.

The main effect of rs6609257 in this study showed that carriers of the A allele had higher VSWM brain activity in fronto-parieto-occipital brain regions. There was no significant age $\times$ genotype interaction, although visual inspection of the data suggested a stronger age-related increase in brain activity individuals with an $\mathrm{A}$ allele, in particular for males. Because of its genomic localization, $\sim 6.6 \mathrm{~kb}$ downstream of the MAOA gene and $\sim 13 \mathrm{~kb}$ upstream of the neighboring $M A O B$ gene, one cannot exclude that rs6609257 also has an effect on $M A O B$. Informatics analysis in the UCSC Genome Browser (hg18 assembly) showed that rs6609257 is located in a genomic region devoid of annotated transcripts. It is currently unknown whether there are any functional promoters or enhancers in this region affecting the brain. However, it is of modest evolutionary conservation and it does not overlap with predicted RNA secondary structures typical for non-coding
RNA (EvoFold annotation). Therefore, it is unlikely that the region contains undiscovered coding elements.

To our knowledge, rs6609257 is a genetic marker that has not previously been associated with VSWM activity or capacity. However, it potentially tags the previously mentioned functional MAOA VNTR 30-bp repeat. The high-activity variant of the VNTR has been associated with higher enzyme expression and lower amine concentration, ${ }^{55}$ as well as increased prefrontal brain activity ${ }^{44-46,48,49}$ and lower levels of aggression. ${ }^{54}$ Our results suggest that the $A$ allele of rs6609257 may act in a similar way for VSWM brain activation. Because information about VNTR repeats was not available for our participants, CEU population (Caucasians) data from the International Hapmap Project (http://hapmap.ncbi.nlm.nih.gov/) were examined for linkage disequilibrium between a SNP $3 \mathrm{~kb}$ distant from the VNTR (rs4570308) and rs6609257. The SNP rs6609257 is on the far end of a major linkage disequilibrium block spanning the MAOA gene region also including the VNTR and rs4570308 markers. The two SNPs are in high but not complete linkage disequilibrium $\left(D^{\prime}=0.84\right)$. However, as the allele frequencies in our total study population and the HapMap CEU population are similar (HapMap: A allele $=60 \%, G$ allele $=40 \%$; Brainchild: A allele $=59 \%, \quad$ G allele $=41 \%$ ), rs6609257 or any other informative SNP located in MAOA could potentially act as a surrogate for the tandem repeat. $^{52}$ Our results require replication to answer this question and future studies on MAOA and VSWM could benefit from examining potential haplotypes including both VNTR and SNP polymorphisms.

Theoretically, X-linked genes have an increased likelihood of sex-specific functions. However, in vivo research indicates that MAOA and MAOB protein levels in the human brain are the same for both sexes. ${ }^{56,57}$ Likewise, our results indicated a minimal influence of sex on outcome. However, when data were split into separate groups for males and females, the genetic effect on VSWM brain activity was significant only for males and at trend-level for females. Interestingly, we were unable to detect an effect of the COMT Val158Met polymorphism (rs4680) on ROI brain activity, contrary to earlier findings using a voxel-based approach in participants 18 years or younger, ${ }^{22}$ or in adult populations. ${ }^{58}$ However, the lack of COMT rs4680 findings here are likely to reflect differences in methodology, as our previous study ${ }^{22}$ revealed small parietal and frontal clusters showing an effect of genotype, whereas the current study focused on larger ROI analyses, based on the broad network of brain regions activated during VSWM.

The fMRI findings from this longitudinal study are in line with cross-sectional VSWM fMRI findings showing that increased activation in fronto-parieto-occipital regions is linked to VSWM performance. ${ }^{15,16,59-61}$ The observed non-linear increase in regional brain activation likely reflects functional maturation, ${ }^{15,16,62}$ and could, speculatively, be an indicator of functional specialization (that is, a relative increase in domain-specific neural activity) of relevant task-related brain regions. To address this, future studies could, for example, compare regional brain activation across multiple cognitive paradigms in a longitudinal developmental sample. Furthermore, the fronto-parieto-occipital regions highlighted in our study are part of the dorsal attention network, which is 
essential for goal-directed (top-down) attention. ${ }^{63}$ Other regions, including bilateral caudate, inferior frontal gyrus/ sulcus, pre-SMA and middle frontal gyrus showed a moderate increase of VSWM brain activation with age, but were not related to VSWM capacity when age was removed as a factor from the model. It is possible, however, that using a higher WM load during scanning might have resulted in an association of BOLD values and capacity also in these regions.

In our study, genetic effects for rs6609257 were strongest on the level of brain activation and did not directly affect VSWM capacity or behavioral problems. This indicates a higher sensitivity of neuroimaging measures to genotypic variation, relative to behavioral measures. Polymorphisms could, however, contribute indirectly to behavioral problems by altering neuromolecular properties required for optimal VSWM function. Cognitive endophenotypes, such as VSWM, may partially mediate such effects, but this requires further investigation. The present study included a community sample with low problem behavior variability and did not reveal direct associations between genotypes of DA system genes and problem behavior. We believe future work in populations with (a high-risk for) psychiatric disorders may be more sensitive to such effects. In sum, the findings from our study provide evidence that VSWM brain activity measures could be used as a powerful phenotype for linking MAO genes to problem behavior in the general population.

\section{Conflict of interest}

The authors declare no conflict of interest.

Acknowledgements. This study was supported by the Knut and Alice Wallenberg Foundation, The Swedish Research Council and a Swedish Royal Bank Tercentennial Foundation Grant in the program 'Learning and Memory in Children and Young Adults' to TK and JK. TZ was supported by a COFAS Marie Curie Fellowship. The funding organizations had no further role in the design and conduct of the study; collection, management, analysis and interpretation of the data; preparation, review, or approval of the manuscript. We thank Jens Gisselgard, Ylva Samuelsson, Douglas Sjöwall and Sissela Bergman Nutley for help with study administration; Kerstin Eriksson and Tomas Jonsson for scanning; and the Mutation Analysis core Facility (MAF) at the Karolinska Institute.

1. Baddeley A. Working memory. Science 1992; 255: 556-559.

2. Conway AR, Kane MJ, Engle RW. Working memory capacity and its relation to general intelligence. Trends Cogn Sci 2003; 7: 547-552.

3. Alloway TP, Gathercole SE, Kirkwood H, Elliott J. The cognitive and behavioral characteristics of children with low working memory. Child Dev 2009; 80: 606-621.

4. Martinussen R, Hayden J, Hogg-Johnson S, Tannock R. A meta-analysis of working memory impairments in children with attention-deficit/hyperactivity disorder. J Am Acad Child Adolesc Psychiatry 2005; 44: 377-384.

5. Goldman-Rakic PS, Selemon LD. Functional and anatomical aspects of prefrontal pathology in schizophrenia. Schizophr Bull 1997; 23: 437-458.

6. Chronis-Tuscano A, Molina BS, Pelham WE, Applegate B, Dahlke A, Overmyer M et al. Very early predictors of adolescent depression and suicide attempts in children with attention-deficit/hyperactivity disorder. Arch Gen Psychiatry 2010; 67: 1044-1051

7. Pantelis C, Yucel M, Bora E, Fornito A, Testa R, Brewer WJ et al. Neurobiological markers of illness onset in psychosis and schizophrenia: the search for a moving target. Neuropsychol Rev 2009; 19: 385-398.

8. Schmeichel BJ, Volokhov RN, Demaree HA. Working memory capacity and the selfregulation of emotional expression and experience. J Pers Soc Psychol 2008; 95: $1526-1540$.

9. Eisenberg N, Valiente C, Eggum ND. Self-regulation and school readiness. Early Educ Dev 2010; 21: 681-698.
10. Klingberg T, Fernell E, Olesen PJ, Johnson M, Gustafsson P, Dahlstrom K et al. Computerized training of working memory in children with $A D H D-$ a randomized, controlled trial. J Am Acad Child Adolesc Psychiatry 2005; 44: 177-186.

11. Gottesman II, Gould TD. The endophenotype concept in psychiatry: etymology and strategic intentions. Am J Psychiatry 2003; 160: 636-645.

12. de Geus EJ, Wright MJ, Martin NG, Boomsma DI. Genetics of brain function and cognition Behav Genet 2001; 31: 489-495.

13. Hariri AR, Weinberger DR. Imaging genomics. Br Med Bull 2003; 65: 259-270.

14. Klingberg T. Training and plasticity of working memory. Trends Cogn Sci 2010; 14: 317-324.

15. Klingberg $\mathrm{T}$, Forssberg $\mathrm{H}$, Westerberg $\mathrm{H}$. Increased brain activity in frontal and parietal cortex underlies the development of visuospatial working memory capacity during childhood. J Cogn Neurosci 2002; 14: 1-10.

16. Kwon H, Reiss AL, Menon V. Neural basis of protracted developmental changes in visuo-spatial working memory. Proc Natl Acad Sci USA 2002; 99: 13336-13341.

17. Olesen PJ, Macoveanu J, Tegner J, Klingberg T. Brain activity related to working memory and distraction in children and adults. Cereb Cortex 2007; 17: 1047-1054.

18. Scherf KS, Sweeney JA, Luna B. Brain basis of developmental change in visuospatial working memory. J Cogn Neurosci 2006; 18: 1045-1058.

19. Thomas KM, King SW, Franzen PL, Welsh TF, Berkowitz AL, Noll DC et al. A developmental functional MRI study of spatial working memory. Neuroimage 1999; 10(3 Pt 1): 327-338

20. Curtis CE, D'Esposito M. The effects of prefrontal lesions on working memory performance and theory. Cogn Affect Behav Neurosci 2004; 4: 528-539.

21. Pessoa L, Gutierrez E, Bandettini P, Ungerleider L. Neural correlates of visual working memory: fMRI amplitude predicts task performance. Neuron 2002; 35 : 975-987.

22. Dumontheil I, Roggeman C, Ziermans T, Peyrard-Janvid M, Matsson H, Kere $\mathrm{J}$ et al. Influence of the COMT genotype on working memory and brain activity changes during development. Biol Psychiatry 2011; 70: 222-229.

23. Söderqvist S, McNab F, Peyrard-Janvid M, Matsson H, Humphreys K, Kere $\mathrm{J}$ et al. The SNAP25 gene is linked to working memory capacity and maturation of the posterior cingulate cortex during childhood. Biol Psychiatry 2010; 68: 1120-1125.

24. Muller U, von Cramon DY, Pollmann S. D1- versus D2-receptor modulation of visuospatial working memory in humans. J Neurosci 1998; 18: 2720-2728.

25. Sawaguchi T, Goldman-Rakic PS. D1 dopamine receptors in prefrontal cortex: involvement in working memory. Science 1991; 251: 947-950.

26. Wahlstrom D, White T, Hooper CJ, Vrshek-Schallhorn S, Oetting WS, Brott MJ et al. Variations in the catechol O-methyltransferase polymorphism and prefrontally guided behaviors in adolescents. Biol Psychiatry 2007; 61: 626-632.

27. Tomasi D, Volkow ND, Wang GJ, Wang R, Telang F, Caparelli EC et al. Methylphenidate enhances brain activation and deactivation responses to visual attention and working memory tasks in healthy controls. Neuroimage 2011; 54: 3101-3110.

28. McNab F, Varrone A, Farde L, Jucaite A, Bystritsky P, Forssberg $\mathrm{H}$ et al. Changes in cortical dopamine D1 receptor binding associated with cognitive training. Science 2009; 323: 800-802.

29. Nemoda Z, Szekely A, Sasvari-Szekely M. Psychopathological aspects of dopaminergic gene polymorphisms in adolescence and young adulthood. Neurosci Biobehav Rev 2011; 35: $1665-1682$

30. Meyer-Lindenberg A, Weinberger DR. Intermediate phenotypes and genetic mechanisms of psychiatric disorders. Nat Rev Neurosci 2006; 7: 818-827.

31. Achenbach TM, Howell CT, Quay HC, Conners CK. National survey of problems and competencies among four- to sixteen-year-olds: parents' reports for normative and clinical samples. Monogr Soc Res Child Dev 1991; 56: 1-131.

32. Agnes Brunnekreef J, De Sonneville LM, Althaus M, Minderaa RB, Oldehinkel AJ, Verhulst FC et al. Information processing profiles of internalizing and externalizing behavior problems: evidence from a population-based sample of preadolescents. J Child Psychol Psychiatry 2007; 48: 185-193.

33. Scott J, Martin G, Welham J, Bor W, Najman J, O'Callaghan M et al. Psychopathology during childhood and adolescence predicts delusional-like experiences in adults: a 21-year birth cohort study. Am J Psychiatry 2009; 166: 567-574.

34. Brett MAJ, Valabregue R, Poline J. Region of interest analysis using an SPM toolbox [abstract]. Presented at the 8th International Conference on Functional Mapping of the Human Brain, June 2-6, 2002, Sendai, Japan, Available on CD-ROM in Neurolmage, Vol 16, No 2. 2002

35. Van Essen DC. A population-average, landmark- and surface-based (PALS) atlas of human cerebral cortex. Neuroimage 2005; 28: 635-662.

36. Van Essen DC, Dierker DL. Surface-based and probabilistic atlases of primate cerebral cortex. Neuron 2007; 56: 209-225.

37. Van Essen DC, Drury HA, Dickson J, Harwell J, Hanlon D, Anderson CH. An integrated software suite for surface-based analyses of cerebral cortex. J Am Med Inform Assoc 2001; 8: 443-459.

38. Alloway TP. Automated Working Memory Assessment Manual. Harcourt: Oxford, UK, 2007.

39. Larsson B, Frisk M. Social competence and emotional/behaviour problems in $6 \pm 16$ yearold Swedish school children. Eur Child Adolesc Psychiatry 1999; 8: 24-33. 
40. Molenberghs $\mathrm{G}$, Verbeke $\mathrm{G}$. A review on linear mixed models for longitudinal data, possibly subject to dropout. Stat Model 2001; 1: 235-269.

41. Field A. Discovering statistics using SPSS. SAGE Publications: London, 2005

42. Akaike H. A new look at the statistical model identification. IEEE Trans Automat Contr 1974; 19: 716-723.

43. Shih JC, Chen K, Ridd MJ. Role of MAO A and B in neurotransmitter metabolism and behavior. Pol J Pharmacol 1999; 51: 25-29.

44. Meyer-Lindenberg A, Buckholtz JW, Kolachana B, Hariri AR, Pezawas L, Blasi G et al. Neural mechanisms of genetic risk for impulsivity and violence in humans. Proc Natl Acad Sci USA 2006; 103: 6269-6274.

45. Passamonti L, Fera F, Magariello A, Cerasa A, Gioia MC, Muglia M et al. Monoamine oxidase-a genetic variations influence brain activity associated with inhibitory control: new insight into the neural correlates of impulsivity. Biol Psychiatry 2006; 59: 334-340.

46. Fan J, Fossella J, Sommer T, Wu Y, Posner Ml. Mapping the genetic variation of executive attention onto brain activity. Proc Natl Acad Sci USA 2003; 100: 7406-7411.

47. Buckholtz JW, Callicott JH, Kolachana B, Hariri AR, Goldberg TE, Genderson M et al. Genetic variation in MAOA modulates ventromedial prefrontal circuitry mediating individual differences in human personality. Mol Psychiatry 2008; 13: 313-324.

48. Cerasa A, Gioia MC, Fera F, Passamonti L, Liguori M, Lanza $P$ et al. Ventro-latera prefrontal activity during working memory is modulated by MAO A genetic variation. Brain Res 2008; 1201: 114-121.

49. Rommelse NN, Altink ME, Arias-Vasquez A, Buschgens CJ, Fliers E, Faraone SV et al. Differential association between MAOA, ADHD and neuropsychological functioning in boys and girls. Am J Med Genet B Neuropsychiatr Genet 2008; 147B: 1524-1530.

50. Zhang J, Chen Y, Zhang K, Yang H, Sun Y, Fang Y et al. A cis-phase interaction study of genetic variants within the MAOA gene in major depressive disorder. Biol Psychiatry 2010; 68: $795-800$.

51. McDermott R, Tingley D, Cowden J, Frazzetto G, Johnson DD. Monoamine oxidase A gene (MAOA) predicts behavioral aggression following provocation. Proc Natl Acad Sci USA 2009; 106: 2118-2123.

52. Craig IW, Halton KE. Genetics of human aggressive behaviour. Hum Genet 2009; 126 $101-113$.
53. Huang SY, Lin MT, Lin WW, Huang CC, Shy MJ, Lu RB. Association of monoamine oxidase $\mathrm{A}$ (MAOA) polymorphisms and clinical subgroups of major depressive disorders in the Han Chinese population. World J Biol Psychiatry 2009; 10(4 Pt 2): 544-551.

54. Buckholtz JW, Meyer-Lindenberg A. MAOA and the neurogenetic architecture of human aggression. Trends Neurosci 2008; 31: 120-129.

55. Sabol SZ, Hu S, Hamer D. A functional polymorphism in the monoamine oxidase A gene promoter. Hum Genet 1998; 103: 273-279.

56. Meyer JH, Ginovart N, Boovariwala A, Sagrati S, Hussey D, Garcia A et al. Elevated monoamine oxidase a levels in the brain: an explanation for the monoamine imbalance of major depression. Arch Gen Psychiatry 2006; 63: 1209-1216.

57. Saura J, Andres N, Andrade C, Ojuel J, Eriksson K, Mahy N. Biphasic and region-specific MAO-B response to aging in normal human brain. Neurobiol Aging 1997; 18: 497-507.

58. Mier D, Kirsch $P$, Meyer-Lindenberg A. Neural substrates of pleiotropic action of genetic variation in COMT: a meta-analysis. Mol Psychiatry 2010; 15: 918-927.

59. Todd JJ, Marois R. Capacity limit of visual short-term memory in human posterior parietal cortex. Nature 2004; 428: 751-754.

60. Mayer JS, Bittner RA, Nikolic D, Bledowski C, Goebel R, Linden DE. Common neural substrates for visual working memory and attention. Neuroimage 2007; 36: 441-453.

61. Olesen P. Combined analysis of DTI and fMRI data reveals a joint maturation of white and grey matter in a fronto-parietal network. Cognitive Brain Res 2003; 18: 48-57.

62. Dosenbach NU, Nardos B, Cohen AL, Fair DA, Power JD, Church JA et al. Prediction of individual brain maturity using fMRI. Science 2010; 329: 1358-1361.

63. Corbetta M, Shulman GL. Control of goal-directed and stimulus-driven attention in the brain. Nat Rev Neurosci 2002; 3: 201-215.

Translational Psychiatry is an open-access journal published by Nature Publishing Group. This work is licensed under the Creative Commons Attribution-Noncommercial-No Derivative Works 3.0 Unported License. To view a copy of this license, visit http://creativecommons.org/licenses/by-nc-nd/3.0/

\section{Supplementary Information accompanies the paper on the Translational Psychiatry website (http://www.nature.com/tp)}

